\title{
ON NONEXTENSIVE STATISTICS, CHAOS AND FRACTAL STRINGS
}

\author{
C.Castro \\ Center for Theoretical Studies of Physical Systems \\ Clark Atlanta University, Atlanta, GA. 30314, USA \\ February 2003, Revised February 2004
}

\begin{abstract}
Motivated by the growing evidence of universality and chaos in QFT and string theory, we study the Tsallis non-extensive statistics (with a non-additive $q$-entropy) of an ensemble of fractal strings and branes of different dimensionalities. Non-equilibrium systems with complex dynamics in stationary states may exhibit large fluctuations of intensive quantities which are described in terms of generalized statistics. Tsallis statistics is a particular representative of such class. The non-extensive entropy and probability distribution of a canonical ensemble of fractal strings and branes is studied in terms of their dimensional spectrum which leads to a natural upper cutoff in energy and establishes a direct correlation among dimensions, energy and temperature. The absolute zero temperature (Kelvin) corresponds to zero dimensions (energy) and an infinite temperature corresponds to infinite dimensions. In the concluding remarks some applications of fractal statistics, quasi-particles, knot theory, quantum groups and number theory are briefly discussed within the framework of fractal strings and branes.
\end{abstract}

\section{1- Introduction}

\subsection{Universality and Chaos in QFT and String Theory}

It has been known for some time that classical Yang-Mills theories exhibit chaotic behaviour [ 1 ] . Spatially varying non-abelian gauge fields on the lattice have revealed that the gauge field has positive Lyapunov exponent that grows linearly with the energy density. Another signal of chaos emerges in the fluctuation properties of the eigenvalues of the staggered Dirac matrix operator in a lattice $S U(3)$ gauge theory ( and in full QCD ). Such fluctuations are described by Random Matrix Theory ( RMT ) [ 1 ] . The nearest-neighbor spacing distribution of the eigenvalues, the distribution of spacings $s$ between two adjacent eigenvalues, agrees with the Wigner distribution for the RMT unitary ensemble : $P(s) \sim s^{2} e^{-4 s^{2} / \pi}$. It has been argued that this could be an indication of quantum chaos.

Many special chaotic solutions of Einstein's equations have also been found [ 37 ]. In particular, a perpetual oscillating chaotic behavior in the vicinity of a spacelike singularity has been found that has the character of deterministic chaos. Chaotica like behaviour has been observed also by [38]. Most recently it has been shown by Polyakov and Kogan [3] that in certain superstring backgrounds involving ghost-matter mixing brane-like vertex operators, the world-sheet dilaton beta function becomes stochastic. The Renormalization Group ( $\mathrm{RG}$ ) equation leads to a non-Markovian Fokker-Planck equation whose solutions are described in terms of the Feigenbaum universal constant $\delta=4.669$.. describing the transition from order to chaos. The appearance of this constant is related to the scaling of spacetime curvatures at the fixed points of the RG flow. Near the singularities, regions where the curvature is very large, the RG flow becomes chaotic. These ghost-matter mixing contributions, behave roughly speaking like the noise terms in the Fokker-Planck equation and can lead to the emergence of chaos near curvature singularities.

Beck [4] has also found a very similar stochastic behaviour for the interaction energy and the potential in his analysis of coupled map lattices. He modeled the vacuum fluctuations on a small ( quantum gravity ) scale as a deterministic chaotic process in terms of iterated maps using Tchebyscheff polynomials ( Ulam maps ). This model was based on earlier work by Kaneko on coupled map lattices (chaotic strings) [6] . These so-called chaotic strings provide the background noise generation for the Parisi-Wu stochastic quantization of ordinary strings and QFT. Extensive numerical evidence, up to 3-5 digits, was presented by Beck that shows that the vacuum energy (the interaction energy) of these chaotic strings is minimized (is zero) precisely for the numerical values of all of the observed Standard Model parameters. Concrete predictions for the vacuum 
expectation values of dilaton-like fields, living on the two-dim lattice, give the values of all the masses, couplings, mixing angles, Higgs mass, mesonic and baryonic resonances, the GUT scale ..of the Standard model. Also numerical values for neutrino masses were obtained.

Other physical models exist that predict the values of couplings and masses, like the Bit-String Physics model based on a Mersenne prime hierarchy $\left(M_{p}=2^{p}-1\right.$, where $p=$ prime $)$ that was developed in the 1970's by Noyes and collaborators [41]; The models based on set theory, hyperdiamond lattices, Clifford algebras and the Feynman Chess board model [42] furnish the values of all the coupling constants and masses with remarkable precision; models based on Scale Relativity [24] and Physical Geometry [23 ] also yield very accurate predictions.

The Parisi-Wu Stochastic quantization, in a sense, establishes a correspondence, or duality, between a classical theory in $D+1$ dimensions, like the bulk of Anti de Sitter space, with a Quantum Theory in $D$-dimensions, like the conformal-projective ( holographic ) boundary of Anti de Sitter space. The ParisiWu fictitious time coordinate plays the role of an extra dimension. Hence one has in this picture a sort of classical/quantum duality. It has been argued recently that the quantized non-abelian gauge theory can be obtained as the infrared limit of the corresponding classical gauge theory in higher dimensions [ 2 ] .

This idea had been exploited earlier on by' t Hooft [2] by arguing that Quantum Mechanics may arise as the low energy limit of a microscopically-deterministic but dissipative theory [2] . Microscopic black hole formation may be the mechanism that causes the loss of information, in the same way that chaos, or exponential growth of sensitivity to initial conditions, causes information loss in Yang-Mills theories [1,2] . Based on the universality and chaos in QFT, and the ubiquitous presence of the stochastic RG equation for the dilaton beta function, as an example, it is reasonable to suggest that there must be a deeper organizing principle (from small to large scales) operating in Nature which might be based in the theories of complexity, nonlinear dynamics and information theory where dimensions, energy and information are intricately connected.

The natural physical setting where this is very likely to occur is in the study of Quantum Field Theories defined in the so-called fractal theory spaces ( a self similar lattice ) which are invariant under the geometrical Renormalization Group transformations [ 7 ]. In the continuum limit, these models described physics in fractal dimensions $D=4+\epsilon$. QFTs defined in these fractal theory spaces could be perturbative finite to all orders in perturbation theory using the standard' t Hooft-Veltman dimensional regularization prescription [7] . Perhaps one of the most peculiar features of QFT's defined in fractal theory spaces, which is connected to the scaling behaviour of fractal strings ( branes ) $[10,16]$, is the plausible existence of limit cycles for the couplings $g^{2}[7]$, regions where the couplings oscillate between large and small values. Wilson long ago had envison this interesting possibility and quite recently this behaviour was observed in superconductivity and the massive sine-Gordon model by [ 43 ]. The latter authors have found a Russian Doll Renormalization Group behaviour where the coupling constants exhibit a cyclical flow and the mass spectrum a hierarchical scaling .

\subsection{Non-extensive Statistics}

Having discussed the relevance of chaos in QFT, Yang-Mills, Gravity, Standard Model and Strings, we will outline briefly some physical applications of Nonextensive Statistics before moving on into the next sections. By non-extensive entropy one means a non-additive entropy : the entropy of a composite system is not equal to the sum of the entropy of its components. The Tsallis non-extensive entropy expression [11] was studied earlier by Havrda and Charvat in a different context [12, 40 ]. It is defined by :

$$
S_{q}=\frac{1-\sum_{j=1}^{N}\left(p_{j}\right)^{q}}{q-1}=\frac{\sum_{j=1}^{j=N} p_{j}\left(1-\left(p_{j}\right)^{q-1}\right)}{q-1} \geq 0
$$

the entropic exponent $q$ is a parameter that can take arbitrary values.

The probabilities of the physical microstates are $p_{j}$, and the summation is performed over all $N$ microstates. A salient feature of the q-entropy $S_{q}$ is that it obeys the non-additive composition rule :

$$
S_{q}^{A+B}=S_{q}^{A}+S_{q}^{B}-(q-1) S_{q}^{A} S_{q}^{B}
$$


and for this reason one refers to non-extensive statistics [ 11, 45 ]. In the $q=1$ case, after using L' Hopital's rule, one reproduces the Boltzmann-Gibbs-Shannon additive entropy .

The onset of chaos associated with the non-linear logistic map have revealed very profound connections among the entropic index $q$, the fractal dimension of the chaotic attractor, the non-linearity exponent, and the end points of the multi-fractal singularity spectrum $f(\alpha),(\alpha$ are the Lipschitz-Holder exponents ) $[9,29,30]$. The emergence of chaos in Renormalization-Group ( RG ) flows has recently been analysed by [ 50]. Chaos of Yang-Mills theories within the context of M-theory ( Matrix theory ) was studied by [ 25] . For these reasons we believe that the Renormalization Group ( RG ) transformations should be extended to the study of fractal strings and p-branes [10]. Since this is an arduous task, we shall concentrate for the time being in the study of the non-extensive Statistical properties of the recently constructed fractal strings and p-branes [10] within the context of the Tsallis entropy $[11,12]$.

This non-extensive entropy formalism of fractal strings is more general than previous ones based on the Boltzmann-Gibbs-Shannon entropy, and also admits a thermodynamics interpretation. Mendes-France has given a Boltzmann-Gibbs-Shannon like definition of the entropy of a ( planar ) curve in terms of the average number of intersection points of the curve with a given random line. In particular, with a random vertical and horizontal array of lines [13]. A Heisenberg-like uncertainty principle applied to chaotic curves in the sense that it is not possible to locate precisely a point on a curve together with the direction of its tangent.

Non-equilibrium systems with complex dynamics in stationary states may exhibit large fluctuations over long time intervals of intensive quantities like temperature, chemical potential, energy dissipation [17] . There are many different statistical descriptions depending on the statistical nature of the fluctuation properties. Tsallis statistics [45] is one of them but other classes exist as well that have been named superstatistics [17] . The most important feature of these different statistical descriptions is that for small variance of the fluctuations all of them behave in a universal way. It seems that the ergodic hypothesis of ordinary Statistical Mechanics (that trajectories cover uniformly all of phase space ) is violated in many physical processes which nevertheless follow the principle of Tsallis non-extensive statistics. The latter non-extensive Statistics has applications in many areas of physics like in turbulence, nuclear high energy physics and cosmic rays astrophysics [17] ; anomalous diffusion of quarks in quark-gluon plasma, the solar neutrino problem [36], the identification of the edge of quantum chaos [3 ], nonlinear fractional Fokker-Planck equation [34], selfsimilar time series [33] , etc...

It is very suggestive to use also Tsallis statistics to examine the spatial and thermal fluctuations in Black Hole thermodynamics if one notices that the Hawking temperature of Black Hole evaporation in four dimensions is inversely proportional to the Black Hole mass $T \sim(1 / M)$, meaning that $\beta \sim M$, and consequently, the mass content, or energy of the Black Hole is proportional to the square of the coordinates fluctuations, like it occurs in an ordinary oscillator. The logarithmic corrections to the Black Hole AreaEntropy relation were obtained by using a collections of extended oscillators in Clifford spaces by [18] and by other methods [44] and the Hawking mass-temperature relationship was obtained as well. For a recent detailed study of the applications of the non-extensive entropy to examine the thermodynamic properties of Self-Gravitating systems of stellar systems see [19, 20] . Non-extensive statistics seem to be very suitable also in studying the spacetime quantum fluctuations as a source of noise which may be related to the so-called Wheeler's spacetime foam at the Planck scale. The spacetime foam could be a natural model for the noise terms in a Parisi-Wu Stochastic-like quantization approach to gravity.

Studies of criticality in non-linear one-dimensional maps have corroborated the validity of Tsallis nonextensive statistics at the critical points . Exact RG fixed-point maps convey the physical meaning of the universal and non-extensive entropy maxima [ 9 ]. More closely related to the work on fractal strings and branes is the fractal-inspired statistical model within the context of Tsallis statistics studied by Oprisal [46] . The purpose of this work is to explore further the applications of non-extensive statistics and fractals within the framework of fractal strings and branes.

The outline of this work is the following. In section $\mathbf{2}$ we present a brief review of the fractal strings and branes ( sprays ) construction described by Lapidus-Frankenhuysen [ 16 ] and focus mainly on two very specific examples. We explain why the dimensions of these fractal strings and sprays are complex valued in general that is intricately linked with the notion of logarithmic periodicty phenomena in many branches of science. In section $\mathbf{3}$ the non-extensive entropy and probability distribution associated with a canonical ensemble of fractal strings ( branes ) is evaluated explicitly in terms of the spectral-energies . It 
then permits us to show why a natural upper cutof $f$ energy exists in this ensemble of fractal strings ( branes ), establishing a direct correlation among dimensions, energy and temperature. In the concluding remarks some applications of fractal statistics, quasi-particles of intermediate statistics, knot theory, quantum groups and number theory are discussed within the framework of fractal strings and branes.

\section{Fractal Strings and Branes}

In this section we will present a very concise review of Fractal Srings and Branes. This review is indispensable to the reader before one can move on to section $\mathbf{3}$ where we study the non-extensive statistics of an ensemble of fractal strings and branes and arrive at the important relationship among dimensions, energy and temperature.

\section{1 Fractal strings}

We will briefly summarize the basic ideas behind the book by [16 ] on Fractal strings. A standard fractal string $\mathcal{L}$ is a bounded open subset $\Omega$ of the real line $R$. It is well known to the experts that such a set consists of countably many open intervals, the lengths of which will be denoted by $l_{1}, l_{2}, l_{3} \ldots . l_{j}$. These are called the lengths of the string. The sum $\sum l_{j}$ is finite and equals the Lebesgue measure of $\Omega$. Despite the fact that one is dealing with countably many intervals/lengths in the definition of $\Omega$ the boundary $\partial \Omega$ is not necessarily countably finite. The boundary of the Cantor string is the ternary Cantor set which is a non countable dust of points (it has the same cardinality as the real line) despite having zero measure.

The geometric counting function of the lengths is defined as:

$$
\zeta_{\mathcal{L}}(s)=\sum_{j=1}^{\infty}\left(l_{j}\right)^{s} .
$$

The central notion of the book [16 ] is that this function of $s$ has poles at complex numbers $s_{n}$, the socalled complex dimensions of a fractal string. The spectrum of a fractal string consists of the sequence of frequencies: $f=k / l_{j}$ for $k, j=1,2,3 \ldots$. The spectral zeta function of the fractal string $\mathcal{L}$ is defined as:

$$
\zeta_{\nu}(s)=\sum_{f} f^{-s}
$$

The geometry and the spectrum of $\mathcal{L}$ are connected by the following relationship, valid for all values of $s$ :

$$
\zeta_{\nu}(s)=\zeta_{\mathcal{L}}(s) \zeta_{R}(s)
$$

where the Riemann zeta function is:

$$
\zeta_{R}(s)=\sum n^{-s}
$$

it has a simple pole at $s=1$ with residue 1 and it admits an analytic continuation to the whole complex plane.

The Cantor string is defined by constructing the sequence of lengths using two scaling factors $r_{1}=r_{2}=$ $1 / 3$. One starts with the unit interval and scales it by these two scaling factors yielding two segments of length equal to $1 / 3$. One then repeats this process iteratively yielding the Cantor string which is a self similar string consisting of segments of lengths $3^{-n}$ with multiplicities of $1,2,4, \ldots 2^{n}$ respectively. The geometric counting function of the Cantor string is then given by the geometric series:

$$
\zeta_{C S}(s)=\sum 2^{n} 3^{-n s}=\frac{1}{1-23^{-s}}
$$

The complex dimensions are given by the poles of this function, by the zeros of the denominator:

$$
23^{-s}=1=1 e^{i 2 \pi n} .
$$


Taking logarithms on both sides of this equation yields:

$$
\ln 2-\operatorname{sln} 3=0 \pm i 2 \pi n \Rightarrow s=\frac{\ln 2}{\ln 3} \pm \frac{i 2 \pi n}{\ln 3} .
$$

we immediately can recognize that the real part of the complex dimension $(\ln 2 / \ln 3)$ coincides precisely with the fractal dimension of the ternary Cantor set. The boundary of the fractal string $\mathcal{L}$ is precisely the uncountable Cantor fractal dust of points.

There are many types of fractal strings discussed in the book [16 ]. The possibilities are endless. In order not to confuse the reader with the nomenclature used in [16 ] related to the class of Golden and Fibonnaci strings, we should emphasize that our construction of Cantorian-Fractal spacetime [10] is based entirely in a particular class of fractal strings, and higher dimensional fractal sprays or branes, whose fractal dimensions are given by suitable binary powers and pinary powers of the Golden mean. By pinary we mean powers of a prime number $p$ :

$$
2^{-\phi^{j}}, \quad p^{-\phi^{j}} . \quad j= \pm 1, \pm 2, \pm 3 \ldots
$$

The complex dimensions of the fractal strings [16 ] is not just a mathematical artifact but that is deeply related to the log-periodic laws in Nature discussed amply by Nottale et al [14 ] in their theories of the Fractal Tree of life and Fractal Evolution and by many others in particle physics in the renormalization group context. We believe that quantum gravitational phenomena should involve interference of complex dimensions. With this preamble of some of the basic ideas on fractal strings of the book [16 ] we turn attention to the construction of the notion of fractal sprays (branes) which are the higher dimensional analogs of strings.

\section{2 . Fractal Branes/Sprays}

In this section we are going to generalize the construction of fractal strings to the $p$-branes case. In particular we will be studying a subclass of fractal branes called sprays in the literature [16 ]. A self-similar fractal brane ( spray ) $\Omega$ with basic shape $B$, scaled by a self-similar string $\mathcal{L}$, whose dimension can be greater than one, is given by a collection of sets $\Omega_{j}$ which are congruent to $l_{j} B$, the homothetic of $\Omega$ by the ratio $l_{j}<1$ for each value of $j$.

Hence, for instance, the fractal spray of the Cantor string on the unit square $B$ is obtained by having one open unit square; two open squares of length-size $1 / 3$, four open squares of length-size $1 / 9 \ldots$ and so forth. The Sierpinski drum is also a fractal spray associated with the unit area triangle $\mathcal{T}$ and is obtained by scaling the middle triangle with the scaling ratios: $r_{1}=r_{2}=r_{3}=1 / 2$. Notice now that $\sum r_{j}=3 / 2>1$ and for this reason the dimension of this fractal spray will be greater than unity. There is one triangle of unit area, 3 triangles of $1 / 4$ area, 9 triangles of $1 / 16$ area, and so forth.

The geometric length counting function associated with the scaling lengths $r_{1}=r_{2}=r_{3}=1 / 2$ of the Sierpinski drum is:

$$
\zeta_{\mathcal{L}}(s)=\sum\left(l_{j}\right)^{s}=\sum 3^{n} 2^{-n s}=\frac{1}{1-32^{-s}} .
$$

and has poles at:

$$
s=\frac{\ln 3}{\ln 2} \pm \frac{i 2 \pi n}{\ln 2} .
$$

Notice that the real part of $s$ is given by $D=(\ln 3 / \ln 2)>1$ which is precisely the fractal dimension of the Sierpinski's gasket. For this reason $D$ is also the dimension of the boundary of the fractal spray $\Omega$ associated with the string $\mathcal{L}$.

Hence we have that:

$$
d-1 \leq D=\operatorname{dim}(\partial \Omega) \leq d \Rightarrow 1 \leq \frac{\ln 3}{\ln 2} \leq 2 .
$$

where $d=2$ is the dimension of the ambient embedding space $R^{2}$ used in the construction of the fractal spray $\Omega$.

Now we are ready to discuss the relevant examples that we shall be studying in the next section when we evaluate the non-extensive entropy of an ensemble of fractal strings and branes. In particular, the two 
cases we shall investigate are the Golden Fractal strings ( branes ) whose fractal dimensions are related to the Golden Mean, and the Fractional strings ( branes ) whose dimensions are related to suitable binary powers with negative and positive integer exponents, respectively.

The construction of Golden fractal strings and sprays ( branes ) in the ambient space $R^{D}$ allowed us to show why Cantorian-Fractal spacetime $\mathcal{E}^{(\infty)}[8]$ comprised of an infinite hierarchy of sets $\mathcal{E}^{(j)}$ of dimension:

$$
(\phi)^{j-1} \cdot(1+\phi)^{j-1} \cdot \quad j>1 .
$$

is a very special class of fractal strings and sprays [10 ] whose scaling ratios are given by suitable binary ( pinary ) powers of the Golden mean $2^{-(1+\phi)^{j-1}}$ and $2^{-\phi^{j-1}}$ respectively. These fractal strings and sprays involving the Golden mean must not be confused with the Golden strings discussed by the authors [16 ]. The latter Golden strings belong to the class of nonlattice self-similar strings, whereas the former belong to the class of lattice self-similar strings.

Let us consider the Golden fractal spray $\Omega$ obtained by scaling an open square $B$ of unit area by the scaling lengths:

$$
r_{1}=r_{2}=2^{-\phi}
$$

Thus $\Omega$ is a bounded open subset of $R^{2}$ consisting of one open square of unit area, 2 open squares of lengthsize $2^{-\phi}$ (area is $2^{-2 \phi}$ ), 4 open squares of length size $2^{-2 \phi}$ (area is $2^{-4 \phi}$ ), etc... The geometric length counting function associated with the scaling: $r_{1}=r_{2}=2^{-\phi}$ is:

$$
\zeta_{\mathcal{L}}(s)=\sum\left(l_{j}\right)^{s}=\sum 2^{n} 2^{-n \phi s}=\frac{1}{1-22^{-\phi s}}
$$

and it has poles when the denominator vanishes:

$$
\begin{gathered}
1=1 e^{i 2 \pi n}=22^{-\phi s} \Rightarrow 0 \pm i 2 \pi n=\ln 2-\phi \operatorname{sln} 2 \Rightarrow \\
s=(1+\phi) \pm \frac{i 2 \pi n(1+\phi)}{\ln 2}
\end{gathered}
$$

where we have used the defining relation of the Golden Mean:

$$
\frac{1}{\phi}=1+\phi \Rightarrow 1=\phi+\phi^{2} \Rightarrow \phi=\frac{\sqrt{5}-1}{2}=0.618 \ldots
$$

It is not difficult to prove that the Golden mean generates a ring structure $Z[\tau]$, where $\tau=1+\phi$ :

$$
(1+\phi)^{n}=F_{n+1}+\phi F_{n} . \quad \phi^{n}=(-1)^{n} F_{n-1}+(-1)^{n+1} F_{n} \phi .
$$

where $F_{n}$ are the Fibonacci numbers $1,1,2,3,5,8,13,21 \ldots$ obeying the recursive relation: $F_{n+1}=F_{n}+F_{n-1}$ and the limit of $F_{n+1} / F_{n}=1+\phi$ when $n \rightarrow \infty$. This construction generalizes to the full space $\mathcal{E}^{(\infty)}$ with the provision that one constructs the Golden sprays from suitable hypercubes of unit hypervolumes and of enough dimensionality $N$ to obey:

$$
N>(1+\phi)^{n}=F_{n+1}+\phi F_{n}>N-1 .
$$

For example, to construct the set $\mathcal{E}^{(4)}[8]$ whose dimension is $(1+\phi)^{3}=4+\phi^{3}=3+2 \phi>4$ requires starting with a unit size hypercube in $R^{5}$, instead of $R^{4}$. To construct the set $\mathcal{E}^{(5)}$ whose dimension is $(1+\phi)^{4}=5+3 \phi>6$ requires starting with a unit size hypercube in $R^{7}$. And in general to construct the set $\mathcal{E}^{(j)}$ whose dimension is $(1+\phi)^{j-1}$ requires to find the smallest integers $N(j)$ such that:

$$
N(j)>(1+\phi)^{j-1}=F_{j}+\phi F_{j-1}>N(j)-1 .
$$

and to begin the construction of the Golden spray by starting with a unit size hypercube in $R^{N(j)}$ whose scaling ratios are:

$$
r_{1}=r_{2}=2^{-\phi^{j-1}} . \quad j=2,3,4,5, \ldots
$$


The poles of the corresponding geometric counting length:

$$
\zeta_{\mathcal{L}}(s)=\sum 2^{n} 2^{-n \phi^{j-1} s}=\frac{1}{1-22^{-\phi^{j-1} s}}
$$

are obtained as usual, from the zeros of the denominator:

$$
\begin{gathered}
1=1 e^{i 2 \pi n}=22^{-\phi^{j-1} s} \Rightarrow 0 \pm i 2 \pi n=\ln 2-\phi^{j-1} \operatorname{sln} 2 \Rightarrow \\
s=(1+\phi)^{j-1} \pm \frac{i 2 \pi n(1+\phi)^{j-1}}{\ln 2} \cdot j>1 .
\end{gathered}
$$

Hence, the dimension of the fractal boundary of the domain $\Omega$ living in the ambient space $R^{N(j)}$ coincides precisely with the dimension of the set $\mathcal{E}^{(j)}$ :

$$
N(j)-1 \leq(1+\phi)^{j-1}=\operatorname{dim} \partial(\Omega) \leq N(j)
$$

This procedure also works for fractal strings whose dimensionality is less than one:

$$
0 \leq D \leq 1
$$

This requires starting with the interval $(0,1)$ and constructing a hierarchy of fractal strings of dimensionality:

$$
0 \leq \phi^{j-1} \leq 1 \cdot j>1
$$

by using the scaling ratios $r_{1}=r_{2}=2^{-(1+\phi)^{j-1}}$. The corresponding geometric counting lengths of this hierarchy of fractal strings are:

$$
\zeta_{\mathcal{L}}=\sum 2^{n} 2^{-n(1+\phi)^{j-1} s}=\frac{1}{1-22^{-(1+\phi)^{j-1} s}} .
$$

whose poles are located at:

$$
s=\phi^{j-1} \pm \frac{i 2 \pi n \phi^{j-1}}{\ln 2} \cdot j>1 .
$$

The dimensions of this hierarchy of fractal strings is $0 \leq D \leq 1$

We could have built another class of Golden fractal strings and sprays by using the scaling factors given respectively by powers of other primes :

$$
p^{-(1+\phi)^{j-1}} \cdot p^{-\phi^{j-1}} \cdot \quad p=\text { prime. } j>1 .
$$

Taking $p=3$ our basic domain would have been a triangle. Then we scale this triangle by the scaling functions $r_{1}=r_{2}=r_{3}=3^{-\phi}$ to generate 3 congruent triangles of areas $3^{-2 \phi}$ smaller than the original and that are attached symmetrically to the 3 sides of the unit triangle. Repeating this process ad infinitum will yield $9,27, \ldots 3^{n}$.. scaled-down versions of the unit triangle and allows to construct a model of the set $\mathcal{E}^{(2)}$ as well. The geometric counting function will be:

$$
\zeta_{\mathcal{T}}(s)=\sum p^{n} p^{-n \phi^{j-1} s}=\frac{1}{1-p p^{-\phi^{j-1} s}} .
$$

and whose poles furnish the complex dimensions at:

$$
s=(1+\phi)^{j-1} \pm \frac{i 2 \pi n(1+\phi)^{j-1}}{\ln p} . \text { Golden sprays. } j>1 .
$$

The Golden fractal strings have for complex dimensions :

$$
s=(\phi)^{j-1} \pm \frac{i 2 \pi n(\phi)^{j-1}}{\ln p} . \text { Golden strings } j>1 .
$$


The real part of the dimension coincides again with the dimension of the set $\mathcal{E}^{(j)}=(1+\phi)^{j-1}$. Only the periods of the imaginary components will vary accordingly to different values of $p=2,3,5,7, \ldots$. It is essential to use scalings given by powers of primes: pinary powers. This construction of these fractal sprays from the scalings of the unit size domain, and their subsequent iterated attachings, resembles nothing but a crystal growth process; i.e and assembly of hypersurfaces.

So far we have been discussing scaling ratios in terms of suitable binary ( pinary) powers and whose exponents are given in terms of the Golden Mean. However, among the infinite number of possible fractal strings and sprays, we could have built yet another interesting class of fractal strings and sprays, which we shall label as fractional strings ( since the dimensions are rationals numbers, fractions, instead of irrational numbers ) and sprays, by using different scaling factors whose exponents are given respectively by :

$$
p^{-2^{j-1}} \text { (strings). } \quad p^{-2^{-(j-1)}} \text { (sprays). } j>1 . \quad p=\text { prime. }
$$

leading to following complex dimensions for the strings and sprays :

$$
\begin{gathered}
s=(2)^{-(j-1)} \pm \frac{i 2 \pi n(2)^{-(j-1)}}{\ln p} . \quad \text { Fractional strings. } j>1 . \\
\left.s=(2)^{j-1} \pm \frac{i 2 \pi n(2)^{j-1}}{\ln p} . \text { sprays(branes }\right) . j>1 .
\end{gathered}
$$

\subsection{Renormalization Group, Log-periodicity and Complex Dimensions}

To finalize this section we argue how Log-periodicity in Nature is a natural physical basis of complex dimensions [10 ]. In the past years a lot of activity has been concentrated on the Log-periodic laws associated with the fractal structures of evolutionary trees. In particular, in the study of the time sequences of major evolutionary leaps at various time scales [14 ]. Models of this type have been observed in economical crisis patterns in Western pre-Columbian civilizations. The physical model underlying the appearance of such laws is that of critical phenomena. The Renormalization Group approach also predicts both power laws and logarithmic-periodic corrections. The log-periodic behaviour in field theories with RG limit cycles has been studied recently by [43].

If the fractal dimension $D$ is complex valued, $d_{x}+i d_{y}$, then one can see that the imaginary component will be responsible for an oscillatory behavior which yields the logarithmic-periodic character to a general function of the form $[10,14]$ :

$$
\begin{gathered}
L=L_{o}(x)\left(\frac{\epsilon_{o}}{\epsilon}\right)^{i d_{y}}=L_{o}(x) \exp \left[i d_{y} \ln \left(\epsilon_{o} / \epsilon\right)\right] \Rightarrow \\
L=L_{o}(x) \cos \left[d_{y} \ln \left(\frac{\epsilon_{o}}{\epsilon}\right)+\alpha\right] . \quad \alpha=\text { phase. }
\end{gathered}
$$

where in the case of the fractal tree of life description [ 14 ] one has the following identification of the time intervals :

$$
\epsilon_{o}=t_{o}-t_{c} . \quad \epsilon=t-t_{c} .
$$

where $t_{c}$ is the critical time marking the end of an evolutionary process which began at $t_{o}$ and $t$ is the time variable. One can see that the function $L$ has peaks at discrete values of time $t_{n}^{(j)}$ that accelerate towards the critical dates in a given tree-branch $\mathcal{L}_{j}$ parametrized by $j$ according to a log-periodic law :

$$
\frac{t_{n}^{(j)}-t_{c}}{t_{o}-t_{c}}=2^{-n(1+\phi)^{j-1}}<1 .
$$

where in this particular case we have used the complex dimensions of the Golden fractal strings ( sprays).

Setting the phase factor $\alpha$ to zero in (2-34) and using for complex dimensions those associated with the Golden fractal strings we have the following hierarchy of imaginary components of complex dimensions associated with a given tree-branch $\mathcal{L}_{j}$ : 


$$
d_{y}=d_{j}^{(n)}=\frac{2 \pi n}{\ln 2}(\phi)^{j-1}
$$

Thus the arguments of the cosine function ( 2-34) will then be:

$$
\left[\frac{2 \pi(\phi)^{j-1}}{\ln 2}\right] \ln 2^{n(1+\phi)^{j-1}}=\left[(1+\phi)^{j-1} \phi^{j-1}\right][2 \pi n]=2 \pi n . \quad \phi(1+\phi)=1 .
$$

and hence, we will have peaks spaced at log-periodic intervals.

Thus, we can then model the fractal evolutionary tree process of Nottale et al [14] in terms of fractal strings/sprays; i.e. in terms of a sequence of temporal intervals of lengths $t_{n}^{(j)}-t_{c}$ associated with a given branch $\mathcal{L}_{j}$. Hence, the fractal tree of life envision by [14 ] fits very naturally within the context of fractal strings and their complex dimensions described in the book [16 ]. These arguments apply to other physical processes involving log-periodicity in critical phenomena [ 43 ] .

\section{The Tsallis Statistics of Fractal Strings and Branes}

Having presented the preamble of chaos and non-extensive statistics physics in the introduction, and reviewed the construction of fractal strings and sprays ( branes ), in this section we shall evaluate explicitly the non-extensive entropy associated with a fractal string ( branes ) ensemble comprised of an infinity of fractal strings and p-branes. To be precise once more, by fractal strings here we mean fractal in the sense of Lapidus-Frankenhuysen [16] , an object whose boundary has for fractal dimensions those between zero and one, like the Cantor set. Whereas the boundaries of their higher dimensional counterparts, fractal sprays or fractal p-branes, have dimensions greater than one. As we have shown these fractal extended objects have complex valued dimensions and In this respect they may reveal new mathematical and physical structures in the horizon.

The non-extensive entropy valid for non-equilibrium systems with a stationary state :

$$
S_{q}=\frac{1-\sum_{j=1}^{j=N}\left(p_{j}\right)^{q}}{q-1} .
$$

is written in terms of the entropic exponent $q$, the probabilities of the physical microstates $p_{j}$, and the summation is performed over all $N$ microstates. We are using the old conventions of Tsallis to define the entropic index $q$,

One may extremize the entropy with the condition $\sum p_{j}=1$ by introducing a Lagrange multiplier. The entropy $S_{q}$ is then extremized for all values of $q$ in the equi-probability case $p_{j}=1 / N$. It gives in this case the entropy for the microcanonical ensemble [11] :

$$
S_{q}=k_{B} \frac{N^{1-q}-1}{1-q} .
$$

For $q \leq 1$ the entropy $S_{q}$ in eq- ( 3-2 ) diverges in the large $N=\infty$ limit. However, for $q>1$, the entropy ( $3-2$ ) reaches a maximum value in the large $N$ limit :

$$
\lim _{N \rightarrow \infty} S_{q}=\frac{k_{B}}{q-1} .
$$

Having discussed the microcanonical ensemble case, we shall proceed with the construction of the canonical ensemble and evaluate the entropy that is extremized for all values of $q$, with the provision that :

$$
\sum_{j=1} p_{j}=1 . \quad \sum_{j=1} p_{j} E_{j}=E .
$$

Introducing two Lagrange multipliers $\alpha, \beta$ allows to extremize the entropy for all values of $q$ for the following probability distribution :

$$
p_{j}=\frac{\left[1-\beta(q-1) E_{j}\right]^{\frac{1}{q-1}}}{Z_{q}} .
$$


At this stage is important to emphasize that our definition for the probability ( $3-5$ ) is based in the old convention used by Tsallis. A different convention has been adopted afterwards by many authors by simply replacing the $q-1$ term by $-(q-1)=1-q$. In this work we shall be using the old convention of Tsallis given by eq-(3-5 ). This convention is very important to keep in mind in all what follows next.

The q-normalization factor is:

$$
Z_{q}=\sum_{k}\left[1-\beta(q-1) E_{k}\right]^{\frac{1}{q-1}}
$$

where the Lagrange multiplier parameter $\beta$ is identified with the inverse temperature $\beta=\left(1 / k_{B} T\right)$. In the $q=1$ limit one recovers the ordinary Boltzmann-Gibbs expression after a simple application of L' Hopital's rule:

$$
p_{j}=\frac{e^{-\beta E_{j}}}{\sum_{k} e^{-\beta E_{k}}} .
$$

and the Shannon-Boltzmann-Gibbs entopy :

$$
S=-k_{B} \sum_{j} p_{j} \ln p_{j}
$$

by a straightforward use of L' Hopital's rule. In our old conventions, when $q<1$, then $q-1<0$, and the numerator of the probability distribution $p_{j}$ in eq- (3-5) diverges at :

$$
\beta E_{j}=\frac{1}{q-1}
$$

Such case ( 3-9 ) corresponds to negative energies ( to a lower cutoff) and after normalizing $p_{j}$ by the $Z_{q}$ factor, which is also infinite when ( $3-9$ ) occurs, it leads to $p_{j} \rightarrow 1$. In the opposite end, the probability $p_{j}$ vanishes for $\beta E_{j} \rightarrow \infty$.

However, an entire different ( complementary ) behaviour occurs for $q>1$ such that the probability distribution ( $3-5$ ) has an upper cutof $f$ value in energy when the probability $p_{j}$ becomes zero. The upper energy cutoff occurs at :

$$
\beta E_{\text {cutoff }}=\frac{1}{q-1}
$$

which means that the relevant energies $E$ involved in the evaluation of the entropy must have a lower and upper bound :

$$
0 \leq(q-1) E \leq(q-1) E_{\text {cutoff }} \leq k_{B} T . \quad q>1 .
$$

Notice that for $q>1$, this cutoff value $\frac{1}{q-1}$ of the ratio $E_{\text {cutoff } f} / k_{B} T$, also matches the maximum value of the microcanonical ensemble entropy, in units of $k_{B}$, given by eq- ( 3-3) . If the cutoff energy $E_{c}>0$ , the last expression (3-10) has a very important physical consequence as described by Tsallis [11]: the temperature $(1 / \beta)$ in the energy intervals : $0<E<(q-1) E_{c}$ is physically unaccessible, thus generalizing the unaccessibility of the absolute zero temperature in ordinary thermodynamics. In order to physically access the absolute zero temperature it is required that the cutoff energy be precisely equal to zero : $E_{c}=0$ $(q>1)$. However due to the zero point vacuum fluctuations $E_{c}>0$ the absolute zero temperature is unattainable.

Having said this we can obtain the canonical probability distribution of an infinite ensemble of fractal strings (branes) $\mathcal{S}_{1}, \mathcal{S}_{2}, \mathcal{S}_{3}, . . \mathcal{S}_{j}, \ldots$ of different dimensions whose characteristic energies are $E_{1}, E_{2}, E_{3}, . . E_{j} \ldots$ The remaining step is to find the energy spectrum. Quantization of ordinary membranes ( p-branes ) is a extremely difficult task. As far as we know it has not been achieved yet. Let alone fractal ones ! If this is the case, how are we able to compute their spectrum ? A plausible way to tackle this brane quantization problem is by arguing [ 27 ], for example, why a membrane can be seen as a collective excitation of an infinity of strings and recurring to the area-preserving symmetry algebra of membranes studied in [28, 29]. .

To bypass this problem of membrane ( brane ) quantization in [ 10 ] we assigned for the characteristic energies of a fractal string ( each fractal string is itself comprised of an infinity of disjoint segments ) 
those values given directly in terms of their ( complex ) fractal dimension by arguing that the imaginary component of the complex dimensions of the fractal string reflect its dimensional and geometrical oscillations $[10,16]$. For this reason it is very important to emphasize that the energy level excitations we have assigned to a fractal string are not naively equal to integer multiples of the inverse lengths (frequencies) of the infinite line-segments required to built a particular fractal string, whose boundary points is a Cantor set of dimensions $0<\operatorname{Real}(s)<1$. [ 16 ]. They are given instead by integer multiples of the characteristic energy; i.e, by the imaginary parts of the complex dimensions. If we believe that the energy of a fractal string ( brane ) is a measure of dimensions and entropy ( information ), we can show for a very specific example [ 10 ] why the characteristic energies of the Golden fractal strings ( branes ) are proportional to the dimensions of the Cantor sets $\mathcal{E}^{(j)}$ in Cantorian-Fractal spacetime [ 8,10].

Therefore, we shall take for the energies of this particular Golden fractal string ( brane ) ensemble $\mathcal{S}_{j}$ the following [10]:

$$
\begin{gathered}
E_{j}=\left[m \frac{2 \pi}{\ln p}\right](1+\phi)^{-(j-1)}=M(1+\phi)^{-(j-1)}=M \phi^{j-1} \cdot(\text { Golden strings }) . \\
E_{j}=\left[m \frac{2 \pi}{\ln p}\right](1+\phi)^{(j-1)}=M(1+\phi)^{(j-1)} .(\text { Golden sprays }) . j>1 .
\end{gathered}
$$

after defining $M=m \frac{2 \pi}{\ln p}$ where $m$ is a fiducial mass scale.

Whereas the energies of the fractional strings and sprays case are given instead by the following binary powers :

$$
\begin{gathered}
E_{j}=\left[m \frac{2 \pi}{l n p}\right] 2^{-(j-1)}=M 2^{-(j-1)} .(\text { Fractional strings }) \\
E_{j}=\left[m \frac{2 \pi}{\operatorname{lnp}}\right] 2^{(j-1)} .=M 2^{(j-1)}(\text { sprays }) \quad j>1 .
\end{gathered}
$$

The quantity $m$ is a fiducial mass scale that for convenience purposes we take to be independent of parameter $j$. Such binary scaling behaviour for the energies has also been found in the fermion spectrum of the chaotic-strings studied by Beck [4,5 ] . The energy $E_{j}$ thus corresponds to the fundamental dimensionalgeometrical oscillation of the Golden and fractional string ( brane) which is encoded in the imaginary parts of the complex dimensions. $p$ is a prime number. As one varies the values of $j$ one spans the full family of Golden and fractional strings ( branes ) $\mathcal{S}_{j}$ within the ensemble.

One can notice in eqs- ( $3-11,3-12,3-13,3-14)$ the hierarchical scaling dependence of the energies of the Golden and fractional strings ( branes ). Such scaling dependence has also been observed in the gap-energies of an infinite number of condensates in a reduced BCS model of Superconductivity and in the infinite number of resonances of the S-matrix associated with the masive sine-Gordon model which exhibits a cyclic coupling-symmetry [43].

The masses have the so-called Russian Doll scaling behaviour :

$$
m_{n} \sim \frac{1}{L} e^{\frac{n \pi}{\theta}}
$$

and behave under scalings as :

$$
m_{n}\left(e^{-\lambda} L\right) \sim m_{n+2}(L) .
$$

where the characteristic Renormalization Group time (length) scale is $L$ and the RG period is $\lambda=\frac{2 \pi}{\theta}$ where $\theta$ is related to the RG invariant coupling parameter $Q$ given in terms of the two couplings of the massive sine-Gordon model $g_{1}, g_{2}$ by the relation $Q \sim\left(g_{1}\right)^{2}-\left(g_{2}\right)^{2}$ [43]. It was speculated by these authors that the three generations of fermions in the Standard model may in fact be among the first few in an infinite sequence of Russian dolls .... The cyclic RG behaviour may possibly represent a new paradigm in Physics .

Finally, by using eqs- ( 3-11, 3-12 ) as an example one can write the canonical probability distribution of Golden fractal string ( brane ) ensemble explicitly in powers of $(1+\phi)$ since $E_{j} \sim(1+\phi)^{j-1}$ :

$$
p_{j}=\frac{\left[1-\beta(q-1) M(1+\phi)^{j-1}\right]^{\frac{1}{q-1}}}{Z_{q}} .
$$


with the $q$ - normalization factor :

$$
Z_{q}=\sum_{k}\left[1-\beta(q-1) M(1+\phi)^{k}\right]^{\frac{1}{q-1}} .
$$

When $q=1$ one recovers the Gibbs-Bolzman probability after using L' Hopital's rule. $p_{j} \sim e^{-\beta E_{j}}$. As we discussed earlier by using the old convention of Tsallis, when $q<1$, there is no upper bound on the energy and as $E_{j} \rightarrow \infty$, the probability $p_{j}$ in eq-( 3-17) becomes zero. However, when $q>1$, a different behaviour occurs and there is a natural cutoff in energy which corresponds to the point when the probability vanishes. In this case, the upper limit $k$ of the sum $Z_{q}$ is given by the cutoff value $k(q)$ defined by :

$$
\beta E_{k}(\text { cutoff })=\beta M(1+\phi)^{k(q)}=\frac{1}{q-1}
$$

Since the $k$ values correspond to natural numbers, to be more precise one should write for the cutoff ( integer ) values $j_{c}$ such that $(1 / q-1)$ has a lower and upper bound given by :

$$
\beta M(1+\phi)^{j_{c}} \leq \frac{1}{q-1} \leq \beta M(1+\phi)^{j_{c}+1} .
$$

where we have denoted by $j_{c}$ the cutoff value of $j$.

The same result applies to the fractional strings ( sprays ) case by simply replacing the powers $(1+\phi)^{j-1}$ by binary powers $2^{j-1}$. One can notice from these equations above that the greater the temperature is, the higher the cutoff values of $k(q)$ can be, which in turn imply higher values for the allowed dimensions of the fractal strings ( branes ). Hence, dimensions, temperature and energy are explicitly interconnected in this physical model. This is one of the most important conclusions of this work. The entropic exponent $q>1$ is directly related to the cutof $f$ values of the Energies ( dimensions ).

For example, when the $j \rightarrow \infty$ limit, the dimension of the Golden fractal string $(\phi)^{j-1}$ collapses to zero. The same occurs for the fractional string. In this limiting case the cutoff energy becomes zero and corresponds naturally to the absolute zero ( Kelvin ) temperature resulting from eq- ( 3-19), when the entropic exponent $q>1$, when using the old conventions by Tsallis.

In the case of fractal branes ( sprays ) the dimension of the fractal branes ( sprays ) given by $(1+\phi)^{j-1}$ becomes infinity ( instead of zero ), saturating its upper bound. Naturally, the temperature in eq- (3-19) blows up in this case. Ample arguments were given in [31 ] why an upper limiting temperature in Nature is given by the Planck Temperature, which in turn, was associated with a maximal acceleration Relativity principle in phase spaces and with a minimal Planck Scale Relativity [ 30 ] . This upper limiting acceleration and temperature, with a minimal Planck scale, is also tantamount to a maximal string tension principle operating in string theory [31] . Therefore, from now on we shall set an upper limit of $T_{\text {Planck }}$.

To sum up, if we equate $\beta E_{j}$ to their saturation values given in terms of the entropic exponent $q$ by eq- (3-19), and using the old conventions of Tsallis for $q>1$, one obtains the following thermal-energy spectrum of the Golden fractal strings, whose dimensions $\phi^{j}$ are constrained to fall between 0 and 1 , :

$$
\beta E_{j}=\beta M(\phi)^{j_{\text {cutoff }}}=\beta M(1+\phi)^{-j_{\text {cutoff }}}=\frac{1}{q-1} .
$$

From the latter equation one can infer that there is an upper and lower bound in the dimensions and temperatures of the fractal strings given by :

$$
0 \leq(q-1) M \phi^{j} \leq(q-1) M \phi^{j_{c u t o f f}} \leq k_{B} T . \text { Golden strings. }
$$

It is very important to add the following remarks in eq-(3-21). Firstly, we have renamed the exponents $j-1$ appearing in the (fractal) dimensions of the Golden fractal strings ( branes ) by simply writing $j$. Secondly, since our conventions for the Golden Mean are $\phi=(1+\phi)^{-1}=0.618<1$, by the cutoff value $j_{\text {cutoff }}$ in eq-(3-21b) one means a lower value in $j$, instead of an upper value, because if $j_{c} \rightarrow \infty$ then $\phi^{j_{c}} \rightarrow 0$, the fractal string collapses to a point.

Whereas the spectrum of fractal branes is : 


$$
\beta E_{j}=\beta M(1+\phi)^{j_{c u t o f f}}=\frac{1}{q-1}
$$

From which one can infer that there is an upper and lower bounds in the dimensions/temperatures of fractal branes ( sprays ) given by:

$$
1 \leq(q-1) M(1+\phi)^{j} \leq(q-1) M(1+\phi)^{j_{\text {cutoff }}} \leq k_{B} T \leq k_{B} T_{\text {Planck. }} \text { Golden sprays (branes) }
$$

where by cutoff $j_{c}$ one means in eq-(3-23) an upper value in $j$ because $1+\phi=1.618>1$.

In the fractional strings case where powers of the Golden mean are replaced by binary powers we have the following conditions for the thermal-energy spectrum of the fractional strings :

$$
0 \leq(q-1) M 2^{-j} \leq(q-1) M 2^{-j_{\text {cutoff }}} \leq k_{B} T . \text { Fractional strings } .
$$

where by cutoff $j_{c}$ in eq-(3-24) one means a lower value in $j$.

And for branes ( sprays ) one has :

$$
1 \leq(q-1) M 2^{j} \leq(q-1) M 2^{j_{\text {cutoff }}} \leq k_{B} T \leq k_{B} T_{\text {Planck. }} . \text { sprays (branes). }
$$

where by cutoff $j_{c}$ in eq-(3-25) one means an upper value in $j$.

Concluding, for each given fixed value of the entropic exponent $q>1$, using the old conventions of Tsallis for the values of $q$, one has arrived in these last equations $(3-21,3-22,3-23,3-24,3-25)$ at the very specific relation constraining the values of the temperatures $k_{B} T=(1 / \beta)$ in terms of the spectral energy levels and dimensions of fractal strings ( branes ) via the cutoff values of the exponents $j_{c}$. These dimensions/energies/temperatures interplay in fractal strings ( branes ) is consistent with the fact that the energy of a fractal string ( brane ) is directly linked to the imaginary part of its dimension; namely, with its dimensional-geometrical oscillations, and must not be confused with the frequencies associated with the infinite number of segments ( domains ) in the construction of a particular string ( brane ) [ 16 ] . The latter authors have shown as well that one can in fact hear the shape of a fractal string as a result of these dimensional-geometrical oscillations.

\section{Concluding Remarks}

We have seen how the study of fractal strings and sprays ( branes ) of complex dimensions, in conjunction with the Tsallis non-extensive statistics, leads to the very important fact that dimensions, energy and temperature are deeply interconnected. Using the old convention of Tsallis, when the entropic exponent $q<1$, there is no upper bound on the energy, while the $q>1$ case introduces an upper cutoff in the energy and dimensions of the fractal string ( brane ) that is related to the temperature. A dimension-temperature relation has been recently studied in RG flows, phase transitions and critical phenomena in spaces of continuous dimensions [52 ]. A multi-fractal generalization of ordinary fractal strings and sprays has not ben developed yet, to our knowledge. The statistics of multi-fractal systems within the framework of Renyi entropy has been studied in $[39,40]$ and non-classical additive entropies by [ 45$]$. It is an open problem how to extend this construction to the multi-fractal strings ( sprays ) case.

Another relevant issue is the role of Quantum groups and the connection between the quantum deformation parameter and the entropic exponent $q$ in the Tsallis statistics. The spectral behaviour of models with Quantum Group symmetries, and fractal geometry, with a geometric-like growth of their eigenvalues like the models studied in this work, has been studied further in [48] . Not surprsingly this should have important applications to the work on fractal strings and sprays ( branes ). Closely related to this is its relation with the fractal spin statistics, the fractal index and the Knot invariants associated with the quantum paths of quasi-particles ( fractons ) in spacetime with fractal Hausdorff dimensions [ 15 ].

There are several different statistical descriptions of quasi-particles and anyons in two-dimensions. In particular, the statistics for quasi- particles of continous spin based on the generalized Fibonnaci series has been studied in [ 51 ] ; the statistics for quasi-particles coined fractons with fractal spin has been developed 
by da Cruz [ 15 ] ; the so-called detailed-balanced statistics models associated with particles of intermediate statistics between bosons and fermions was presented in [ 49 ]; and the fractal inspired statistics model within the context of Tsallis statistics studied earlier by Oprisal [46 ]. It is warranted to find a unifying picture, if possible, of all these different statistical descriptions associated with quasi-particles of continous spin ( fractal spin ) and with intermediate statistics.

The new idea of thermal duality in string thermodynamics under the transformations $T$ goes to $1 / T$ has been advanced in [ 47 ]. This was achieved by extending the usual definition of entropy and other thermodynamical quantities while implementing the invariance ( covariance ) under thermal duality symmetry transformations of the thermal string partition function and other thermodynamical quantities. The Tsallis entropy $S_{q}$ is not invariant under the " duality " transformation $q$ goes to $1 / q$. It is important to find what would be the generalization of the Tsallis entropy $S_{q}$ and statistics that implement invariance ( covariance ) under such duality symmetry transformations of the entropic exponents, in a similar vein to the thermal duality symmetry advocated in string thermodynamics [47].

Finally, the chaotic attractors in Renormalization Group flows in Quantum Field Theories [ 50 ] deserves to be studied further within the framework of number theory, arithmetic attractors and preferred CalabiYau compactfications in String theory [53 ]. In particular the relation to the deep underlying physics behind the Standard Model parameters described by Beck [ 4, 5 ] because the moduli space of stringcompactifications determine the particle masses, Yukawa couplings and other GUT model parametes....etc. Hence, it is desirable to figure out how string theory compactifications overlaps with the description in [ 4,5 ] based on chaotic scalar fields and two-dimensional Kaneko coupled-map lattices. A lot remains to be done to link nonlinear dynamical systems with string theory. Hopefully, fractal strings and branes combined with their non-extensive statistics may shed some light into this very difficult question.

\section{Acknowledgements}

We thank Alexander Gorban for insightful discussions and to B.G Sidharth and K.Rama for their very kind invitation and hospitality to the Birla Science Center ( Hyderabad ) and the Institute of Mathematical Sciences ( Madras ) where this work was completed. To J. Mahecha and M. Altaisky for invaluable assistance and to the referee for important and useful remarks to improve this work.

\section{References}

1- S. Matiyan, G. K. Saviddy and N. G Saviddy, Sov. Phys. JETP 53 ( 1981 ) 421. B. Berg, E. Bittner, H. Markum, R. Pullirsch , M. Lombardo and T. Wettig, "Universality and Chaos in Quantum Field Theories" [ arXiv : hep-lat/0007008] .

2-G.'t Hooft, Class. Quan. Gravity 16 ( 1999 ) 3283. T. Biro, B. Muller and S. Matinyan : " Chaotic quantization : "Maybe the Lord plays dice After all?" [ arXiv : hep-th/0301131] .

3-I. Kogan, D. Polyakov, 'Ghost-Matter Mixing and Feigenbaum Universality in String Theory" [arXiv : hep-th/0212137].

4- C. Beck, "Chaotic Strings and Standard Model Parameters" [arXiv : hep-th/0105152]. Physica 171 D ( 2002 ) 72.

5-C. Beck, Spatio-Temporal Vacuum Fluctuations of Quantized Fields Advances in Nonlinear Dynamics vol. 21 ( World Scientific, Singapore 2000 ) .

6- K. Kaneko, "Spatio Temporal chaos in one and two-dimensional coupled map lattices " Physica 37 D ( 1989 ) 60 .

7- C. Hill, "Quantum Field Theory over a Fractal Theory Space" [arXiv : hep-th/0210076].

8- M. El Naschie, Chaos, Solitons and Fractals 10 ( 2-3 ) ( 1999 ) 567.

9-A. Robledo, "Criticality in non-linear one-dim maps : RG universality map and nonextensive entropy " [arXiv : cond-math/020209].

10- C. Castro, Chaos, Solitons and Fractals 14 ( 2002 ) 1341. C. Castro : Chaos, Solitons and Fractals 15 ( 2003 ) 797. 
11-C. Tsallis, Jour. Of Statistical Physics 52 ( 1988 ) 479.

12- J. Havrda and F. Charvat : Kybernatica 3 ( 1967) 30.

13-M. Mendes-France, "The Planck Constant of a Curve "Fractal Geometry and Analysis, ( 1991 ) 325-366;

J. Belair and S. Dubuc, eds. Kluwer Academic Publishers, Holland.

14-L. Nottale, J. Chaline and P. Grou, " Les arbres de l' Evolution" Hachette Literature, Paris ( 2000 ).

15- W. da Cruz, "Fractal von Neumann Entropy" [arXiv : cond-mat/0201489]. W. da Cruz," Fractons and Fractal Statistics" [arXiv : hep-th/9905229]. W. da Cruz, "The spin-statistics connection, Gauss-Bonnet theorem and the Hausdorff dimension of the quantum paths" [arXiv : hep-th/0402131 ] .

16- M. Lapidus and M. Frankenhuysen, "Fractal strings, complex dimensions and the zeros of the zeta function", Birkhauser, New York ( 2000 ).

17 -C. Beck, Physica 305 A ( 2002 ) 209, [ arXiv : cond-mat/0110007].

18 - C. Castro, A.Granik, Foundations of Physics vol. 33 , No. 3 ( 2003 ) 445.

19- A. Taruya, M. Sakagami, Physica A 307 ( 2002 ) 185.

20- P. Chavanis, "Gravitational instability and polytropic spheres" [arXiv : astro-ph/0207080].

21- C. Castro, A. Granik, Chaos, Solitons and Fractals 12 (2001) 1793.

23- G. Gonzalez-Martin, "Physical Geometry" University of Simon Bolivar, publishers, Caracas, June 2000, 265 pages, ISBN : 9800767495. G. Gonzalex-Martin, "The proton/electron Geometric Mass Ratio" [ arXiv : physics/0009052 ] . G. Gonzalez-Martin, "The fine structure constant from Relativistic Groups" [ arXiv : physics/0009051 ]

24- L. Nottale, Chaos, Solitons and Fractals 16 ( 2003 ) 539.

25- I. Arefeva, P. Mevdevdev, O. Rytchkov ans I. Volovich, Chaos, Solitons and Fractals 10 ( 1999 ) 225.

27 - C. Castro, Chaos, Solitons and Fractals 7 ( 1996 ) 711.

28- A. Cappelli and G. Zemba, Nuc. Phys. B 490 ( 1997 ) 595.

29-X. Shen, " $W_{\infty}$ algebras and String Theory" Int. J. Mod. Phys. A 7 ( 1992 ) 6953.

30-. L. Nottale, Fractal Spacetime and Microphysics , towards Scale Relativity World Scientific , 1992. L. Nottale, La Relativite dans tous ses etats Hachette Literature, Paris 1999.

31- C. Castro, Int. Jour. Mod. Phys. A 18 , No. 20 ( 2003 ) 5445-5473.

32-C-Beck, " Generalized statistical mechanics of cosmic rays " [arXiv : cond-mat/0301354].

33-A-Olemski, " Statistical theory of self-similar time series " [arXiv : cond-mat/0210667].

34-E. Lenzi, L. Malacarne, R. Mendes and I. Pedron, " Anomalous diffusion, nonlinear Fokker-Planck equation and solutions "[ arXiv : cond-mat/0208332].

35-Y. Weinstein, S. Lloyd and C. Tsallis, "The Edge of Quantum Chaos" [arXiv : cond-mat/0206039].

36-C-Tsallis, " Non-extensive Statistical mechanics : A brief review of its present status" [arXiv : condmat/0205571].

37- V. Belinski, E. Lifschitz and I. Khalatnikov, Adv. Phys. 19 ( 1970 ) 525.

38- V. Ivashchuk and V. Melvikov, Jour. Math. Phys 41 ( 2000 ) 6341. T. Damour and M. Henneaux : Phys. Rev. Let 85 ( 2000$) 85$.

39- A. Renyi, Probability Theory ( North Holand, Amsterdam 1970 ) .

40- P. Jizba and T. Arimitsu, "The world according to Reny , Thermodynamics of multifractal systems " [arXiv : cond-mat/0207707].

41-P. Noyes, "A Short Introduction to Bit-String Physics" [arXiv : hep-th/9707020].

42-F. Smith, Int. Jour. Theor. Phys. 24 ( 1985 ) 155. Int. Jour. Theor. Phys. 25 ( 1985 ) 355. "From Sets to Quarks" [ arXiv : hep-ph/9708379] and [ CERN CDS EXT-2003-087 ].

43- A. Le Clair, J. Roman and G. Sierra, "Russian Doll Renormalization Group, Kosterlitz-Thouless Flows and the Cyclic sine-Gordon Model " [arXiv : hep-th/0301042]. A. Le Clair, J. Roman and G. Sierra," Log-periodic behavior of finite size effects in field theories with RG limit cycles " [ arXiv : hep-th/0312141 ] 44- S. Carlip, Class. Quant. Grav 17 ( 2000 ) 4175. R. Kaul and P. Majumdar, Phys. Rev. Letters 84 (2000) 5255. R. Kaul, S. K. Rama, "Black Hole Entropy from Spin One Punctures" [gr-qc/0301128].

45-A. Gorban and I. Karlin, Phys. Rev E 67 ( 2003 ) 016104 . A. Gorban, I. Karlin and H. Ottinger : " Non-classical additive entropy functions and their maximizers " ETH-Zurich preprint, June 2002.

46. S.A. Oprisal, J. Physique 7 ( 1997 ) 853-862.

47. K.Dienes and M. Lennek, " Thermal Duality confronts Entropy, A New Approach to String Thermodynamics" [ arXiv : hep-th/0312173 ] . 
48. M. Tierz," Spectral behaviour of Models with a Quantum Group Symmetry " [arXiv : hep-th/0308121] 49- R. Acharya, P. Narayana Swamy, " Detailed Balanced and Intermediate Statistics " [arXiv : quant$\mathrm{ph} / 0308084]$.

50. A.Morozov, A. Niemi," Chaos in RG flows" [ arXiv : hep-th/0304178 ] . 51- M. Rachidi, E. Saidi and J. Zerouaoui, "Fractional Statistics in terms of Generalized Fibonnaci Sequences " [arXiv : hep-th/0109062]

52-H. Ballhausen, " Critical Exponents from Dimensional Variation " [arXiv : hep-th/0310239] .

53-G. Moore, " Les Houches Lectures on Strings and Arithmetic " [ arXiv : hep-th/0401049 ] . 